\title{
Strain Measurement Using Deformable Image Registration
}

\author{
J.A. Weiss ${ }^{1,2}$, A.I. Veress ${ }^{1}$, G.T. Gullberg ${ }^{3}$, N.S. Phatak ${ }^{1,2}$, Q. Sun ${ }^{1,2}$, \\ D. Parker $^{4}$, R.D. Rabbitt ${ }^{1}$ \\ 1 University of Utah, Department of Bioengineering, USA \\ jeff.weiss@utah.edu \\ 2 University of Utah, Scientific Computing and Imaging Institute, USA \\ 3 Lawrence Berkeley National Laboratory, Center for Functional Imaging, USA \\ 4 Utah Center for Advanced Imaging Research, \\ Center for Advanced Medical Technologies, USA
}

The accurate determination of strain in deforming biological tissues is a necessary and important part of experimental investigations in biomechanics. We have developed a method, referred to as Hyperelastic Warping, to combine medical image data with a solid mechanics analysis approach to allow estimation of tissue strains in the absence of detailed information about boundary conditions and in some cases constitutive information. The method makes use of medical image data to provide information about the deforming tissue and thus the strain and stress fields. The mathematical problem is to search through all admissible configurations for the one that minimizes the difference between a transformed template image and a target image collected experimentally. The resulting deformation map is used to determine the strain field. This technique has been applied successfully to determine strain in several biological soft tissues. This paper describes the theory, implementation and validation of the technique for measurement of transmural strains in the left ventricle.

\section{Introduction}

Nonlinear strains in biological tissues and cells are usually measured using fiducial markers (e.g., Holmes et al. (1995) and McCulloch et al. (1989)). In some cases, the $3 \mathrm{D}$ strain can be estimated directly from changes in the distances between groups of markers making up tetrahedral sets. The inherent invasiveness, inhomogeneous strain fields and physical dimensions of the markers can limit the applicability of this technique. This paper describes a non-invasive method to estimate strain fields in biological soft tissues that may be applied in the absence of discrete fiducials or exact information regarding tissue constitutive properties. The method is based on an algorithm 
for deformable image registration termed Hyperelastic Warping (Rabbitt et al. (1995), Veress et al. (2004), Weiss et al. (1998)). The method makes use of the textural information in image data to determine the deformation map between pairs of images that represent distinct states of deformation. Validation of the method in the context of measuring transmural left ventricular strains is presented.

\section{Methods}

\subsection{Finite Deformation Theory}

A Lagrangian reference frame is assumed. A pair of image datasets, the template and target, represents some physical characteristic of the body before and after deformation, respectively. The images have spatially varying scalar intensity fields defined with respect to the reference configuration and denoted by $T$ and $S$, respectively. The deformation map is $\varphi(\mathbf{X})=\mathbf{x}=\mathbf{X}+\mathbf{u}(\mathbf{X})$, where $\mathbf{x}$ are deformed coordinates, $\mathbf{u}(\mathbf{X})$ is the displacement field, and $\mathbf{F}=\partial \boldsymbol{\varphi}(\mathbf{X}) / \partial \mathbf{X}$ is the deformation gradient (Spencer (1980)). The density $\rho$ is related to the Jacobian, $J:=\operatorname{det} \mathbf{F}=\rho_{0} / \rho$, where $\rho_{0}$ is the reference density. It is assumed that $T$ and $S$ have a general dependence on position $\mathbf{X}$ and deformation map $\boldsymbol{\varphi}(\mathrm{X})$. The positive definite, symmetric right Cauchy-Green deformation tensor is $\mathbf{C}=\mathbf{F}^{T} \mathbf{F}$.

\subsection{Variational Framework for Hyperelastic Warping}

Most deformable image registration methods can be posed as the minimization of an energy functional $E$ that consists of at least two terms. The combined energy functional can be defined with respect to the current (deformed) configuration as

$$
E(\mathbf{X}, \boldsymbol{\varphi})=\int_{\mathcal{B}} W(\mathbf{X}, \boldsymbol{\varphi}) \frac{\mathrm{d} v}{J}+\int_{\mathcal{B}} U(T(\mathbf{X}, \boldsymbol{\varphi}), S(\mathbf{X}, \boldsymbol{\varphi})) \frac{\mathrm{d} v}{J}
$$

$W$ is an energy term that provides regularization and/or some type of constraint on the deformation map (e.g., one-to-one mapping or no negative volumes admitted), while $U$ represents an energy that depends on the registration of the image data in the template and target images. $\mathcal{B}$ represents the current volume of integration. In Hyperelastic Warping, a spatial discretization of the template image is deformed into alignment with the target, which remains fixed in the reference configuration. Coordinates of template material points are tracked. In the following presentation, the scalar intensity field of the template, $T$, is not changed directly by the deformation, and thus it is represented as $T(\mathbf{X})$. Since the values of $S$ at material points associated with the deforming template change as the template deforms with respect to the 
target, it is written as $S(\boldsymbol{\varphi})$. The standard formulation uses a Gaussian sensor model:

$$
U(\mathbf{X}, \boldsymbol{\varphi})=\frac{\lambda}{2}(T(\mathbf{X})-S(\boldsymbol{\varphi}))^{2} .
$$

$\lambda$ is a penalty parameter (Reddy (2002)) that enforces the alignment of the images. As $\lambda \rightarrow \infty,(T(\mathbf{X})-S(\varphi))^{2} \rightarrow 0$, and the image energy converges to a finite, minimized value.

It is assumed that $W$, a hyperelastic strain energy, defines the material behavior. It depends on $\mathbf{C}$, which is independent of rotation, and thus hyperelasticity provides an objective (invariant under rotation) constitutive framework, in contrast to linearized elasticity. Equation (1) becomes

$$
E=\int_{\mathcal{B}} W(\mathbf{X}, \mathbf{C}) \frac{\mathrm{d} v}{J}+\int_{\mathcal{B}} U(T(\mathbf{X}), S(\boldsymbol{\varphi})) \frac{\mathrm{d} v}{J} .
$$

The Euler-Lagrange equations are obtained by taking the first variation of $E(\mathbf{X}, \boldsymbol{\varphi})$ with respect to $\boldsymbol{\varphi}$. This can be thought of as a 'virtual displacement' - a small variation in the current coordinates $\mathbf{x}$, denoted $\varepsilon \boldsymbol{\eta}$, where $\varepsilon$ is an infinitesimal scalar. Variations are calculated by taking the Gateaux derivative of $U$ evaluated at with respect to $\boldsymbol{\varphi}+\varepsilon \boldsymbol{\eta}$ and then letting $\varepsilon \rightarrow 0$. The weak form of the Euler-Lagrange equations for (3) is (Simo and Hughes (1998))

$$
G(\boldsymbol{\varphi}, \boldsymbol{\eta}):=D E(\boldsymbol{\varphi}) \cdot \boldsymbol{\eta}=\int_{\mathcal{B}} \boldsymbol{\sigma}: \nabla \boldsymbol{\eta} \mathrm{d} v-\int_{\mathcal{B}} \lambda\left[(T-S) \frac{\partial S}{\partial \boldsymbol{\varphi}} \cdot \boldsymbol{\eta}\right] \frac{\mathrm{d} v}{J}=0 .
$$

Here, $\boldsymbol{\sigma}$ is the second-order symmetric Cauchy stress tensor

$$
\boldsymbol{\sigma}=\frac{1}{J} \mathbf{F} \frac{\partial W}{\partial \mathbf{C}} \mathbf{F}^{T}
$$

Forces applied to the template physical model due to the image data are opposed by internal forces from the deformation of the material through the constitutive model. The form of $W$ depends on the material (Spencer (1980), Weiss et al. (1996)).

Equation (4) is highly nonlinear and an incremental-iterative solution method is necessary to obtain the configuration $\varphi$ that satisfies this equation (Matthies and Strang (1979)). A common approach is based on linearization and iterative solution using Newton's method or some variant. Assuming a solution at configuration $\varphi^{*}$ is known, a solution is sought at some small increment $\varphi^{*}+\Delta \mathbf{u}$. Linearization of (4) at $\varphi^{*}$ in the direction $\Delta \mathbf{u}$ yields

$$
\begin{aligned}
& \mathbf{L}_{\boldsymbol{\varphi}} \cdot G(\boldsymbol{\varphi}, \boldsymbol{\eta})=\int_{\mathcal{B}} \boldsymbol{\sigma}: \nabla \boldsymbol{\eta} \mathrm{d} v-\int_{\mathcal{B}} \lambda\left[(T-S) \frac{\partial S}{\partial \boldsymbol{\varphi}^{*}} \cdot \boldsymbol{\eta}\right] \frac{\mathrm{d} v}{J} \\
& +\int_{\mathcal{B}} \nabla \boldsymbol{\eta}: \boldsymbol{\sigma}: \nabla(\Delta \mathbf{u}) \mathrm{d} v+\int_{\mathcal{B}} \nabla^{s} \boldsymbol{\eta}: \mathbf{c}: \nabla^{s}(\Delta \mathbf{u}) \mathrm{d} v+\int_{\mathcal{B}} \boldsymbol{\eta} \cdot \mathbf{k} \cdot \Delta \mathbf{u} \frac{\mathrm{d} v}{J},
\end{aligned}
$$

where $\mathbf{c}$ is the fourth order spatial elasticity tensor and $\nabla^{s}[\cdot]$ is the symmetric gradient (Bathe (1982)). The first two terms in the second line of equation (6) 
are referred to as the geometric and material stiffnesses, respectively (Bathe (1996)). The third term is the second-order image stiffness for Hyperelastic Warping:

$$
\mathbf{k}=\frac{\partial^{2} U}{\partial \varphi \partial \varphi}=\lambda\left[\left(\frac{\partial S}{\partial \varphi}\right) \otimes\left(\frac{\partial S}{\partial \varphi}\right)-(T-S)\left(\frac{\partial^{2} S}{\partial \varphi \partial \varphi}\right)\right] .
$$

These three terms form the basis for evaluating the relative influence of the image-derived forces and the forces due to internal stresses on the converged solution to the deformable image registration problem.

\subsection{Finite Element Discretization and Solution Procedure}

Hyperelastic Warping is based on a FE discretization of the template. A FE mesh is constructed to correspond to all or part of the template image. $T$ is interpolated to the FE mesh and is convected with the FE mesh. As the FE mesh deforms, the values of $S$ are queried at the current location of the FE mesh nodes. Using Voigt notation (Bathe (1996)), the linearized equations (6) can be written as

$$
\sum_{i=1}^{N_{\text {nodes }}} \sum_{j=1}^{N_{\text {nodes }}}\left(\mathbf{K}^{R}\left(\boldsymbol{\varphi}^{*}\right)+\mathbf{K}^{I}\left(\boldsymbol{\varphi}^{*}\right)\right)_{i j} \Delta \mathbf{u}_{j}=\sum_{i=1}^{N_{\text {nodes }}}\left(\mathbf{F}^{\mathrm{ext}}\left(\boldsymbol{\varphi}^{*}\right)-\mathbf{F}^{\mathrm{int}}\left(\boldsymbol{\varphi}^{*}\right)\right)_{i} .
$$

Equation (8) is a system of linear equations. The term on the left-hand side is the (symmetric) tangent stiffness matrix. $\Delta \mathbf{u}$ is the vector of unknown incremental nodal displacements - for a FE mesh of 8-noded hexahedral elements in three dimensions, $\Delta \mathbf{u}$ has length $8 \times 3 \times N_{\mathrm{el}}$, where $N_{\mathrm{el}}$ is the number of elements in the mesh. $\mathbf{F}^{\text {ext }}$ is the vector of external forces arising from the differences in the image intensity fields, and $\mathbf{F}^{\text {int }}$ is the vector of internal forces resulting from the stress divergence. The material and geometric stiffnesses combine to give the mechanics regularization stiffness $\mathbf{K}^{R}$, and the FE discretization of (7) gives the image-based tangent stiffness $\mathbf{K}^{I}$. An initial estimate of the incremental nodal displacements is obtained by solving (8) for $\Delta \mathbf{u}$ and this solution is improved iteratively using a quasi-Newton method.

In the applications described below, $U$ is treated as a hard constraint, with $W$ providing a regularization. Indeed, the $U$ in equation (2) is essentially a penalty function. The main problem with the penalty method is that as the penalty parameter $\lambda$ is increased, some of the diagonal terms in $\mathbf{K}^{I}$ become very large with respect to others, leading to numerical ill-conditioning. To circumvent this problem, the augmented Lagrangian method is used (Fletcher (1989)). A solution to the governing equations at a particular computational timestep is first obtained with a relatively small penalty parameter $\lambda$. Then the total image-based body forces $\partial U / \partial \varphi$ are incrementally increased in a second iterative loop, resulting in better satisfaction of the constraint. This leads to a stable algorithm that allows the constraint to be satisfied to a user-defined tolerance. Ill conditioning of the stiffness matrix is entirely avoided. 


\section{Application - Strain Measurement in the Left Ventricle}

Assessment of regional heart wall motion (wall motion, thickening, strain, etc.) can identify impairment of cardiac function due to a variety of cardiomyopathies and ischemic myocardial disease. The objectives of this study were to validate the use of Hyperelastic Warping for the extraction of high-resolution strain maps of the left ventricle from cine-MRI images. The sensitivity of predictions to errors in material model selection, material parameter estimation, and simulated noise in the image data was determined. The hypotheses were that (1) Hyperelastic Warping could accurately predict the fiber stretch (final length/initial length along the local fiber direction) and in-plane strain distributions during diastolic (passive) filling from cine-MRI image datasets, (2) variations in the assumed material properties and constitutive model would have a minimal effect on the predicted fiber stretch distribution, and (3) the results of Warping cardiac cine-MRI images would be relatively insensitive to noise in the image data.

\subsection{Methods}

Cardiac Image Acquisition. To mimic the typical imaging procedure used in clinical MR acquisition for patients with cardiac pathologies, gated cine-MRI images of a normal male volunteer's heart (35 years old) were acquired on a 1.5T Siemens scanner using standard clinical resolution and settings $(256 \times 256$ image matrix, $378 \mathrm{~mm}$ FOV, $10 \mathrm{~mm}$ slice thickness, 10 slices) (Fig. 1). The volumetric MRI dataset corresponding to beginning of diastole was designated as the template image. The template image was cropped to $64 \times 64$ image matrix by 8 slices to focus on the heart.

FE Mesh Generation and Boundary Conditions. A conforming FE mesh was used. The boundaries of the LV were obtained by manual segmentation of the epi- and endocardium from the template image (beginning of diastole). The
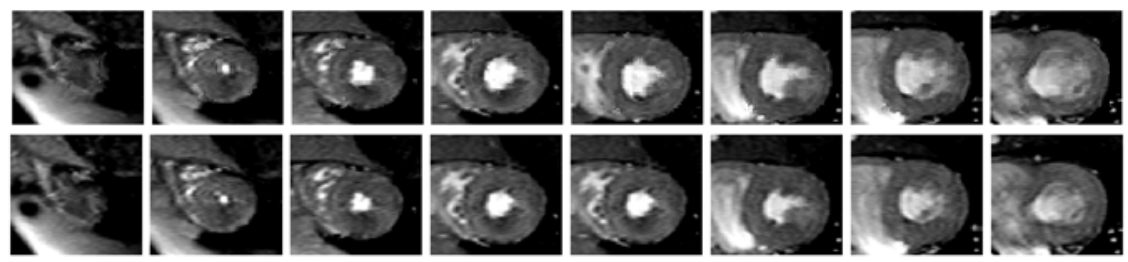

Fig. 1. Target (top) and template (bottom) image datasets used in the Warping analysis. The Target image dataset was created by mapping the template image dataset with displacements determined from a forward FE simulation of passive diastolic filling. 
3D FE model was constructed to include the entire image domain, with the lumen and the tissue surrounding the myocardium represented by a compressible neo-Hookean isotropic hyperelastic constitutive model with relatively soft properties (equivalent linear material coefficients of $E=0.3 \mathrm{kPa}$ and $n=0.3$ ) so that the entire template image could be mapped by the resulting deformation. The edges of the FE mesh were fixed, eliminating rigid body modes.

Constitutive Model and Material Coefficients. The myocardium was represented as a transversely isotropic material with fiber angles varying from $-90^{\circ}$ at the epicardial surface, through $0^{\circ}$ at the mid-wall, to $+90^{\circ}$ at the endocardial surface. The transversely isotropic material model represented fibers embedded in a neo-Hookean matrix with

$$
W=\mu\left(\tilde{I}_{1}-3\right)+F_{2}(\tilde{\lambda})+\frac{\kappa}{2}[\ln (J)]^{2},
$$

where $\tilde{I}_{1}$ is the first deviatoric invariant of the right Cauchy deformation tensor (Spencer (1980)), $\tilde{\lambda}=\sqrt{\mathbf{a}_{0} \cdot \tilde{\mathbf{C}} \cdot \mathbf{a}_{0}}$ is the deviatoric fiber stretch along the local direction $\mathbf{a}_{0}, \mu$ is the shear modulus of the matrix and $\kappa$ is the bulk modulus. The stress-stretch behavior for the fibers was represented as exponential, with no resistance to compressive load:

$$
\begin{array}{ll}
\tilde{\lambda} \frac{\partial F_{2}}{\partial \tilde{\lambda}}=0, & \tilde{\lambda}<1, \\
\tilde{\lambda} \frac{\partial F_{2}}{\partial \tilde{\lambda}}=C_{3}\left[\exp \left(C_{4}(\tilde{\lambda}-1)\right)-1\right], & \tilde{\lambda} \geq 1 .
\end{array}
$$

Here, $C_{3}$ scales the stresses and $C_{4}$ defines the rate of stiffening along the preferred direction. A description of the constitutive model and its FE implementation can be found in Weiss et al. (1996).

Material coefficients were determined by a nonlinear least-squares fit of the constitutive equation to published equibiaxial stress/strain curves (Humphrey et al. $(1990 \mathrm{a}, \mathrm{b}))\left(\mu=2.10 \mathrm{kPa}, C_{3}=0.14 \mathrm{kPa}\right.$, and $\left.C_{4}=22.0\right)$. A bulk modulus $\kappa$ of $160.00 \mathrm{kPa}$ was chosen such that the change in relative volume would remain under $5 \%$, in other words the material would be nearly incompressible. The LV material properties do not need to be exact for this validation analysis because the stretch and strain results from the forward FE model were used as the 'gold standard' for comparison to the Warping results. A physiological internal pressure load was applied to the endocardial surface and a forward nonlinear FE analysis was performed using NIKE3D (Maker et al. (1990)). The forward FE model was validated by comparing predictions of fiber stretch, circumferential, radial and in-plane (radial-circumferential) shear strains, wall thickness, ejection fraction and end-diastolic diameter to values reported in the literature (data not shown).

Creation of Synthetic Target Image. Warping strain predictions were validated by comparing various strain measures against a forward FE simulation in 
which the FE model (based on the template image) was used to generate a synthetic target image. The template and synthetic target image were then input to the warping analysis but with no boundary conditions applied. A synthetic target image was created by applying the displacement map of the forward FE model to the original template MRI image (Fig. 1).

The Warping FE model used the same geometry and material properties as the forward FE model. The Warping analysis was performed using the original template image and the synthetic target image. The nodal values of fiber stretch, circumferential strain, radial strain and shear strain were averaged for each image slice and compared with the values from the forward FE model on a slice-by-slice basis. To assess transmural patterns of deformation, these same measures of deformation were computed as functions of wall position and were then compared with the forward FE predictions. Finally, scatter plots of all nodal values of the four measures of deformation were generated to determine coefficients of determination $\left(R^{2}\right)$ between the Warping and forward FE model predictions.

Sensitivity to Material Coefficients and Constitutive Model. To determine the sensitivity of the Warping analysis to changes in material coefficients, $\mu$ and $C_{3}$ were increased and decreased by $24 \%$ of the baseline values, corresponding to the $95 \%$ confidence interval of the material coefficients reported in Humphrey et al. $(1990 \mathrm{a}, \mathrm{b}))$. To assess the importance of the fiber reinforcement in the constitutive model, the constitutive model was changed to an isotropic neoHookean model for the Warping simulations. Finally, the effect of the material bulk modulus was assessed by increasing and decreasing $\kappa$ by a factor of 10.0 in the Warping analysis. $R^{2}$ values were determined for the four measures of local deformation between the Warping and forward FE model predictions.

Sensitivity to Image Noise. To assess the effects of noise on the Warping predictions of deformation, an additive noise model was used to modify the template and target images. Random noise $n(i, j)$ was added to the images $s(i, j)$, where $i$ and $j$ represent pixel coordinates, to create a noisy image $i(i, j)$ :

$$
i(i, j)=s(i, j)+n(i, j) .
$$

$n(i, j)$ was defined as the standard deviation $\sigma_{N}$ of a zero mean normal probability distribution for noise image intensities (Gonzalez and Woods (1992), pp. 187-213). The signal to noise ratio (SNR) was defined as

$$
\mathrm{SNR}=\frac{\sigma_{I}}{\sigma_{N}}
$$

For the images used, $\sigma_{I}=42$ gray levels. SNRs of $16,8,4,1$ and 0.5 were examined. Local measures of deformation obtained from Warping with the noisy images were compared with results from forward FE predictions to determine the effect of SNR on the $R^{2}$ values. 


\subsection{Results}

Comparison of Forward FE and Warping Predictions. There was good qualitative and quantitative agreement between forward and Warping predictions of fiber stretch (Fig. 2A). The highest values of fiber stretch were near the mid-wall (40-50\% through the wall), decreasing toward the endo- and epicardial surfaces. Fiber stretch was slightly higher at the endocardial surface than the epicardial surface. The magnitudes of circumferential, radial and shear strain were highest at the endocardial wall and decreased toward the epicardial surface (Fig. 2B-D). There was very good agreement between the forward FE and Warping predictions in terms of the magnitudes of strains and their transmural variation for all four measures of local deformation. The average fiber stretch and strains for both the Warping and forward FE results had generally higher standard deviations than the results of each of the individual
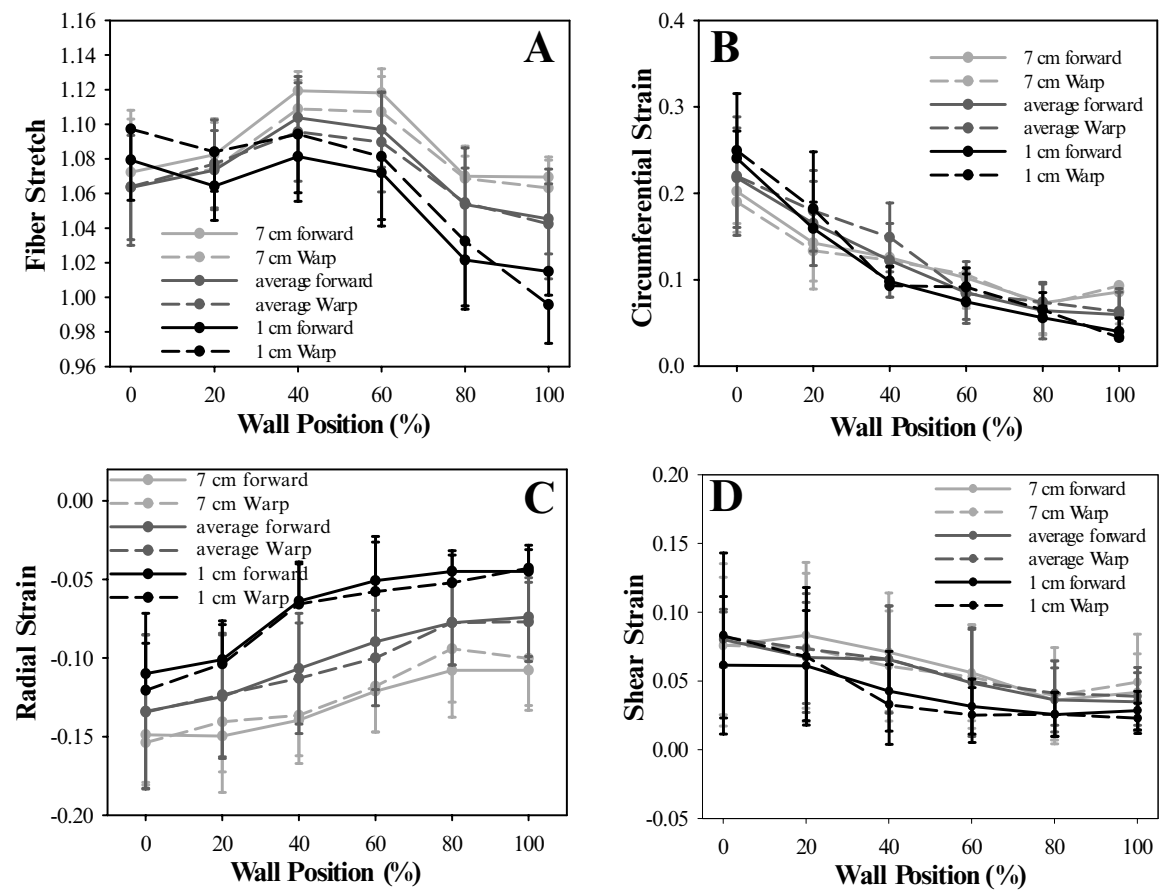

Fig. 2. Forward and FE predictions of local wall deformation at end-diastole as a function of distance through myocardial wall (mean $\pm \mathrm{SD}$ ): A - fiber stretch; $\mathrm{B}$ circumferential Green-Lagrange strain; C - radial Green-Lagrange strain; D - inplane Green-Lagrange shear strain (circumferential/radial). 0\% denotes endocardial and $100 \%$ denotes epicardial. Results are presented for image cross-sectional slices at $1 \mathrm{~cm}$ (black), $7 \mathrm{~cm}$ (light gray) and as an average over all slices (dark grey). $7 \mathrm{~cm}$ corresponds to the base of the LV and $1 \mathrm{~cm}$ is near the apex of the heart. Solid lines are results from forward FE model and dashed lines are results for Hyperelastic Warping. All values are referenced to the undeformed geometry (beginning-diastole). 
image planes (Fig. 2). Thus, the variability depicted in the overall averages is partially due to variability between the results for each image plane. These differences between axial locations are expected because of the changes in LV geometry from apex to base.

Correlation analyses between the forward FE and Warping stretch/strain distributions for each image plane indicated that good agreement was obtained (Fig. 3A-D). There was a significant correlation between the predictions for all four measures of strain ( $p<0.001$ for all cases). The circumferential strain predictions had the best agreement with the forward FE results $\left(R^{2}=0.76\right)$, while the fiber stretch predictions had the lowest coefficient of determination $\left(R^{2}=0.67\right)$.

Sensitivity to Changes in Material Coefficients and Constitutive Model. Warping predictions of fiber stretch and strain were insensitive to changes in material parameters $\mu$ and $C_{3}$ (Table 1 ). The predictions appear to be somewhat sensitive to material model, with the $R^{2}$ values dropping from 1 to 10 points depending on the particular case (Table 1). The technique also appears to be somewhat sensitive to changes in the bulk modulus $\kappa$ of the model depending on whether the material is being made more or less compressible. Increasing the bulk modulus by an order of magnitude resulted in little in-plane strain degradation; however, the stretch results did show a decrease in the $R^{2}$ value.
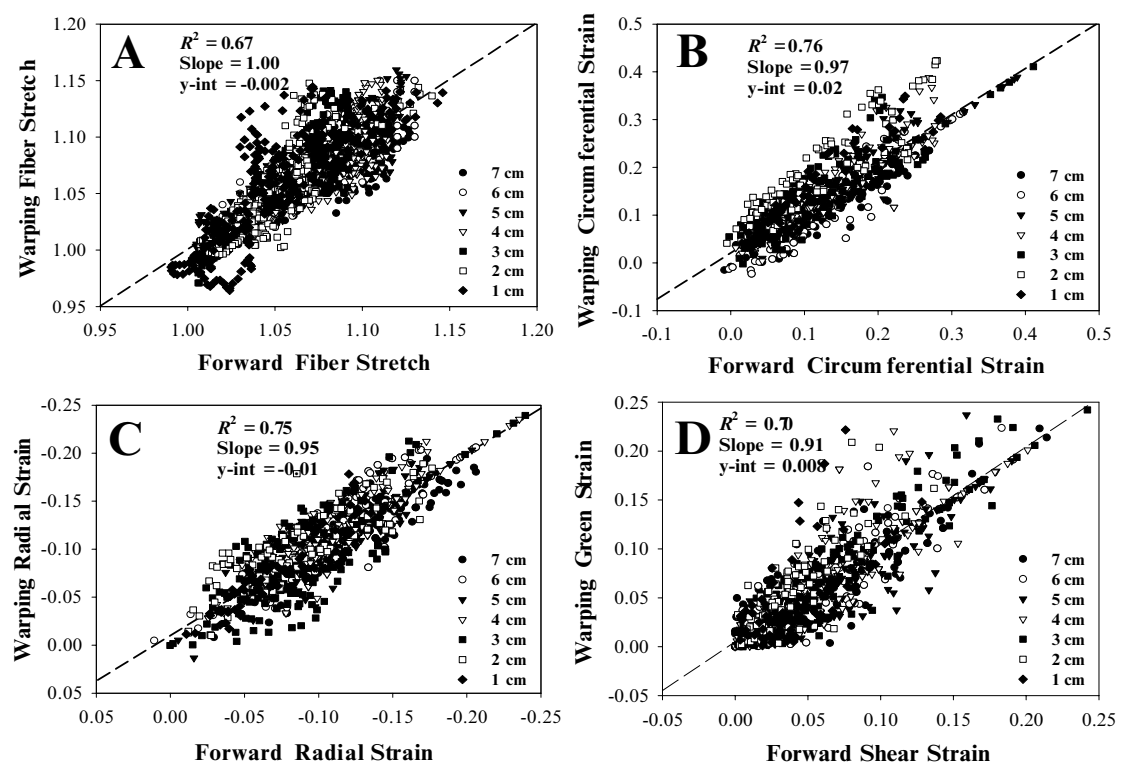

Fig. 3. Scatter plots of forward FE versus Warping stretch/strains: A - fiber stretch; B - circumferential strain; C - radial strain; D - in-plane shear strain. $7 \mathrm{~cm}$ corresponds to the base of the $\mathrm{LV}$ and $1 \mathrm{~cm}$ is near the apex of the heart. 
Table 1. Effect of changes in material coefficients and constitutive model on $\left(R^{2}\right)$ between the Warping and forward FE predictions for the four measures of strain.

\begin{tabular}{c|cccc}
\hline & $\begin{array}{c}\text { Fiber } \\
\text { Stretch }\end{array}$ & $\begin{array}{c}\text { Circ. } \\
\text { Strain }\end{array}$ & $\begin{array}{c}\text { Radial } \\
\text { Strain }\end{array}$ & $\begin{array}{c}\text { Shear } \\
\text { Strain }\end{array}$ \\
\hline Baseline Warping & 0.67 & 0.72 & 0.66 & 0.70 \\
$\mu+24 \%$ & 0.65 & 0.69 & 0.65 & 0.71 \\
$\mu-24 \%$ & 0.64 & 0.70 & 0.65 & 0.70 \\
$C_{3}+24 \%$ & 0.65 & 0.71 & 0.66 & 0.72 \\
$C_{3}-24 \%$ & 0.65 & 0.70 & 0.66 & 0.72 \\
neo-Hookean & 0.57 & 0.65 & 0.58 & 0.69 \\
$\kappa \times 10$ & 0.50 & 0.84 & 0.65 & 0.63 \\
$\kappa / 10$ & 0.55 & 0.40 & 0.30 & 0.27 \\
\hline
\end{tabular}

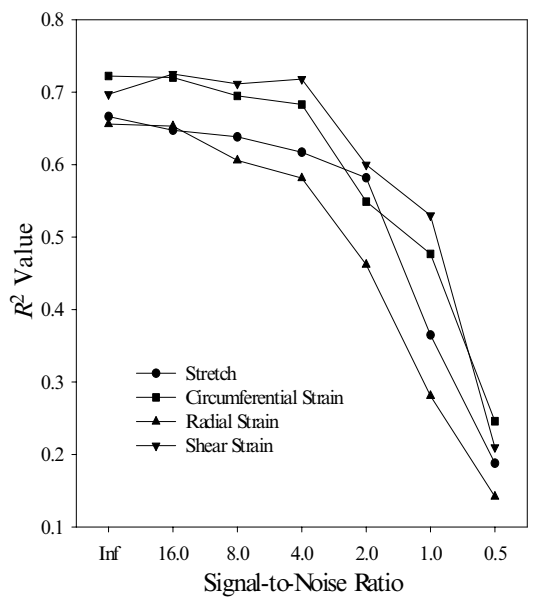

Fig. 4. Effect of signal-to-noise ratio on coefficient of determination.

Decreasing the bulk modulus by an order of magnitude resulted in severe degradation of the results for all measures of deformation.

Sensitivity to Image Noise. There was little change in $R^{2}$ values between the forward FE and Warping predictions down to a SNR of 4.0. Degradation in $R^{2}$ values was progressive for SNRs below 4.0 (Fig. 4).

\section{Discussion}

The results of this study indicate that Hyperelastic Warping can predict strain and fiber stretch distributions of the left ventricle during diastole from clinical cine-MRI images. The material sensitivity studies demonstrated that strain predictions are not highly dependent on the material coefficients used to regularize the registration problem, with the exception that a reasonable estimate 
of the bulk modulus is needed. Warping analysis can accurately determine fiber stretch distribution in relatively noisy images, down to a SNR of 4 . Hyperelastic Warping has the inherent flexibility to analyze images acquired using a variety of modalities (Veress et al. (2003)).

Hyperelastic Warping minimizes the image-based energy in a 'hard' sense using an augmented Lagrangian, while a hyperelastic strain energy regularizes the image registration. This approach is considered to be a major strength of the method. First and foremost, the use of a hyperelastic strain energy in combination with a FE discretization ensures that deformations will be diffeomorphic (one-to-one, onto, and differentiable with a differentiable inverse; see, e.g., Miller et al. (2002). Other image-based techniques that have been used to estimate strains via deformable registration, such as optical flow (Klein et al. (1997)) and texture correlation (Gilchrist et al. (2004)), use only an imagebased minimization term. Thus, these techniques do not ensure physically reasonable deformations in regions that lack image contrast or texture and they are sensitive to noise. Further, there is no guarantee that deformation maps will be diffeomorphic. Second, hyperelasticity is objective for large strains and rotations, while previous solid mechanics-based regularizations were based on linear elasticity (Bajcsy et al. (1983), Davatzikos (1996), Gee et al. (1993)), which is not objective and penalizes finite strains and rotations. Finally, the use of a hyperelastic constitutive model ensures that deformation maps reflect the behavior of an elastic material under finite deformation. In regions of the template model that have large intensity gradients, large image-based forces will be generated and the solution will be primarily determined by the image data. In regions that lack image texture or gradients, image-based forces will be smaller and the predicted deformation will be more dependent on the regularization (material model). A reasonable representation of the material behavior helps to improve predictions in these areas. In the future, more accurate constitutive models should be incorporated to better represent the material properties of the myocardium. Further, more accurate estimates of local fiber direction may be used (Dou et al. (2003)) to further improve the predictions of myocardial strains based on Hyperelastic Warping.

Acknowledgements. Financial support from NSF grant \#BES-0134503, NIH grant \#R01-EB00121, and by the U.S. Department of Energy under contract \#DE-AC03-76SF00098 is gratefully acknowledged. An allocation of computer time was provided by the Center for High Performance Computing at the University of Utah.

\section{References}

Bajcsy, R., Lieberson, R., and Reivich, M. (1983). A computerized system for the elastic matching of deformed radiographic images to idealized atlas images. J. Comput. Ass. Tomography 7:618-625. 
Bathe, K.-J. (1982). Finite Element Procedures in Engineering Analysis. Englewood Cliffs: Prentice Hall.

Bathe, K.-J. (1996). Finite Element Procedures. New Jersey: Prentice Hall.

Davatzikos, C. (1996). Spatial normalization of 3D brain images using deformable models. J. Comput. Ass. Tomography 20:656-665.

Dou, J., Tseng, W. Y., Reese, T. G., and Wedeen, V. J. (2003). Combined diffusion and strain MRI reveals structure and function of human myocardial laminar sheets in vivo. Magn. Reson. Med. 50:107-113.

Fletcher, R. (1989). Practical Methods of Optimization. New Delhi: John Wiley \& Sons.

Gee, J., Reivich, M., and Bajcsy, R. (1993). Elastically deforming 3D atlas to match anatomical brain images. J. Comput. Ass. Tomography 17:225-236.

Gilchrist, C. L., Xia, J. Q., Setton, L. A., and Hsu, E. W. (2004). Highresolution determination of soft tissue deformations using MRI and firstorder texture correlation. IEEE Trans. Med. Imaging 23:546-553.

Gonzalez, R. C., and Woods, R. E. (1992). Digital image processing. Reading, Massachusetts: Addison-Wesley.

Holmes, J. W., Takayama, Y., LeGrice, I., and Covell, J. W. (1995). Depressed regional deformation near anterior papillary muscle. Am. J. Physiol. 269:H262-H270.

Humphrey, J. D., Strumpf, R. K., and Yin, F. C. P. (1990a). Determination of a constitutive relation for passive myocardium: I. A new functional form. J. Biomech. Eng. 112:333-339.

Humphrey, J. D., Strumpf, R. K., and Yin, F. C. P. (1990b). Determination of a constitutive relation for passive myocardium: II. Parameter estimation. J. Biomech. Eng. 112:340-346.

Klein, G. J., Reutter, B. W., and Huesman, R. H. (1997). Non-rigid summing of gated PET via optical flow. IEEE Trans. Nucl. Sci. 44:1509-1512.

Maker, B. N., Ferencz, R. M., and Hallquist, J. O. (1990). NIKE3D: A nonlinear, implicit, three-dimensional finite element code for solid and structural mechanics. Technical report UCRL-MA, Lawrence Livermore National Laboratory.

Matthies, H., and Strang, G. (1979). The solution of nonlinear finite element equations. Int. J. Numer. Meth. Engr. 14:1613-1626.

McCulloch, A. D., Smaill, B. H., and Hunter, P. J. (1989). Regional left ventricular epicardial deformation in the passive dog heart. Circ. Res. 64:721733.

Miller, M. I., Trouvé, A., and Younes, L. (2002). On the metrics and EulerLagrange equations of computational anatomy. Ann. Rev. Biomed. Eng. 4:375-405.

Rabbitt, R. D., Weiss, J. A., Christensen, G. E., and Miller, M. I. (1995). Mapping of hyperelastic deformable templates using the finite element method. SPIE 2573:252-265.

Reddy, J. N. (2002). Energy Principles and Variational Methods in Applied Mechanics. 2nd edition. 
Simo, J. C., and Hughes, T. J. R. (1998). Computational Inelasticity. New York: Springer.

Spencer, A. (1980). Continuum Mechanics. New York: Longman.

Veress, A. I., Weiss, J. A., Gullberg, G. T., Vince, D. G., and Rabbitt, R. D. (2003). Strain measurement in coronary arteries using intravascular ultrasound and deformable images. J. Biomech. Eng. 124:734-741.

Veress, A. I., Phatak, N., and Weiss, J. A. (2004). Deformable image registration with hyperelastic warping. In The Handbook of Medical Image Analysis: Segmentation and Registration Models. New York: Marcel Dekker.

Weiss, J. A., Maker, B. N., and Govindjee, S. (1996). Finite element implementation of incompressible, transversely isotropic hyperelasticity. Comput. Meth. Appl. Mech. Engr. 135:107-128.

Weiss, J. A., Rabbitt, R. D., and Bowden, A. E. (1998). Incorporation of medical image data in finite element models to track strain in soft tissues. SPIE 3254:477-484. 